\title{
TRATAMENTO ARTROSCÓPICO DO IMPACTO FEMOROACETABULAR
}

\author{
ARTHROSCOPIC TREATMENT OF \\ FEMOROACETABULAR IMPINGEMENT
}

\author{
Giancarlo C. Polesello', Marcelo C. Queiroz ${ }^{2}$, Nelson K. Ono ${ }^{3}$, Emerson K. Honda ${ }^{4}$, Rodrigo P. Guimarães ${ }^{5}$, \\ Walter Ricioli Junior ${ }^{6}$
}

\section{RESUMO}

Objetivos: O propósito deste estudo é avaliar os resultados em curto prazo do tratamento artroscópico do impacto femoroacetabular. A hipótese é a de que os resultados do tratamento artroscópico são favoráveis. Métodos: Entre agosto de 2003 e agosto de 2007, 28 quadris foram submetidos ao tratamento do impacto femoroacetabular pela via artroscópica. A idade média dos pacientes foi de 34 anos, com média de seguimento de 27 meses. Quanto à melhora clínica, os pacientes foram avaliados pré e pósoperatoriamente pelo Harris Hip Score (HHS) modificado por Byrd. Os pacientes foram avaliados pré e pós-operatoriamente em relação à rotação interna do quadril acometido. Os valores obtidos nos índices acima foram analisados estatisticamente através do método de Wilcoxon para a avaliação de variáveis não paramétricas. Resultados: O Harris Hip Score médio pré-operatório foi de 54,2 e o pós-operatório, de 94,8 ( $\mathrm{p}<0,001$ ). O aumento médio do HHS foi de 37,5 pontos. Houve quatro resultados bons (15\%) e 24 excelentes (85\%). Pré-operatoriamente os pacientes apresentavam rotação interna do quadril média de $17^{\circ}$ e pósoperatoriamente, de $36^{\circ}$. O aumento médio de rotação interna foi de $19^{\circ}(\mathrm{p}<0,001)$. Conclusão: O tratamento artroscópico do impacto femoroacetabular tem resultados satisfatórios.

Descritores - Osteoartrite do quadril/diagnóstico; Osteoartrite do quadril/etiologia; Osteoartrite do quadril/terapia; Articulação do quadril/patologia; Artroscopia

\section{ABSTRACT}

Objective: The purpose of this study is to evaluate the shortterm follow-up results of arthroscopic treatment of femoroacetabular impingement. Our hypothesis is that arthroscopic treatment results are favorable. Methods: Between August 2003 and August 2007, 28 hips had femoroacetabular impingement treated by hip arthroscopy. The mean age was 34 years, with mean follow-up period of 27 months. Clinical results were graded with the modified Harris hip score, which was measured pre-and postoperatively. Patients had also their internal rotation analyzed. These parameters were calculated by using Wilcoxon's t test for analysis of nonparametric paired samples performed. Results: The mean preoperative Harris Hip Score was 54.2, improving to 94.8 postoperatively $(p<0,001)$. The mean increase was 37.5 points. We had 4 good results (15\%) and 24 excellent results (85\%). Preoperatively, the patients had a mean internal rotation of $17^{\circ}$, and, postoperatively, $36^{\circ}$. The average internal rotation increase was $19^{\circ}(p<0,001)$. Conclusions: The arthroscopic treatment of femoroacetabular impingement presents satisfactory results.

Keywords - Osteoarthritis, hip/diagnosis; Osteoarthritis, hip / etiology Osteoarthritis, hip/therapy; Osteoarthritis, hip/ pathology; Arthroscopy

\footnotetext{
1 - Professor Assistente Doutor da Faculdade de Ciências Médicas da Santa Casa de São Paulo - FCMSCSP. Professor Assistente do Grupo de Quadril - FCMSCSP.

2 - Médico Ortopedista, Ex-Estagiário do Grupo de Quadril da FCMSCSP.

3 - Professor Assistente Doutor da FCMSCSP e Chefe do Grupo de Quadril - FCMSCSP.

4 - Professor Assistente Doutor da FCMSCSP e Membro Sênior do Grupo de Quadril - FCMSCSP.

5 - Professor Instrutor Mestre da FCMSCSP e Assistente do Grupo de Quadril - FCMSCSP.

6 - Médico Ortopedista, Assistente do Grupo de Quadril da FCMSCSP.
} 


\section{INTRODUÇÃO}

Vários fatores podem coexistir na etiologia da artrose coxofemoral, entre eles, anormalidades genéticas, morfológicas e bioquímicas. Embora o mecanismo fisiopatológico do processo degenerativo que afeta o quadril displásico seja bem entendido, muitas artroses "idiopáticas" merecem maior esclarecimento, pois ainda permanecem como a causa mais comum de artrose do quadril. Com base em observações há mais de 40 anos $^{(1)}$, acredita-se que todas as artroses idiopáticas sejam secundarias a sutis alterações acetabulares e femorais previamente subestimadas ou não reconhecidas. Existem evidências crescentes de que o impacto femoroacetabular (IFA) exerça papel importante na etiologia mecânica do desenvolvimento da artrose do quadril ${ }^{(2)}$.

O impacto femoroacetabular é capaz de destruir a articulação do quadril, uma vez que provoca alterações mecânicas articulares que geram ciclo vicioso de ação e reação orgânica. A literatura a esse respeito é unânime nessa afirmação $0^{(3-9)}$.

Diferentemente do joelho, onde as alterações da cartilagem e ligamentares são a causa mais frequente das artroses, no quadril o dismorfismo ósseo, como a displasia e o IFA, leva a lesão do complexo condrolabial e a consequente degeneração articular. $\mathrm{O}$ ortopedista tem a obrigação de reconhecer essas alterações.

A discussão sobre artrose em pacientes jovens até há pouco tempo se limitava em indicar osteotomias, artroplastia ou artrodese, ou seja, atuar após a destruição articular. Com os resultados cirúrgicos favoráveis em curto prazo utilizando-se a técnica aberta ${ }^{(10-12)}$, o tratamento artroscópico tem surgido como nova e promissora opção.

O propósito deste estudo é avaliar os resultados em curto prazo do tratamento artroscópico do impacto femoroacetabular. Nossa hipótese é a de que os resultados do tratamento artroscópico são favoráveis.

\section{MÉTODOS}

Foram selecionados os pacientes submetidos ao tratamento do impacto femoroacetabular com seguimento mínimo de um ano no Grupo de Cirurgia de Quadril do Departamento de Ortopedia e Traumatologia da Faculdade de Ciências Médicas da Santa Casa de São Paulo. Entre agosto de 2003 e agosto de 2007, 36 quadris foram submetidos ao tratamento do impacto femoroacetabular pela via artroscópica. A média de seguimento foi de 27 meses (12 a 60 meses). Todos os pacientes foram reavaliados. Foram excluídos oito pacientes que já apresentavam artrose no exame inicial (Classificação de Tönnis et $a l^{(13)}$ graus 2 e 3 ), totalizando 28 pacientes analisados.

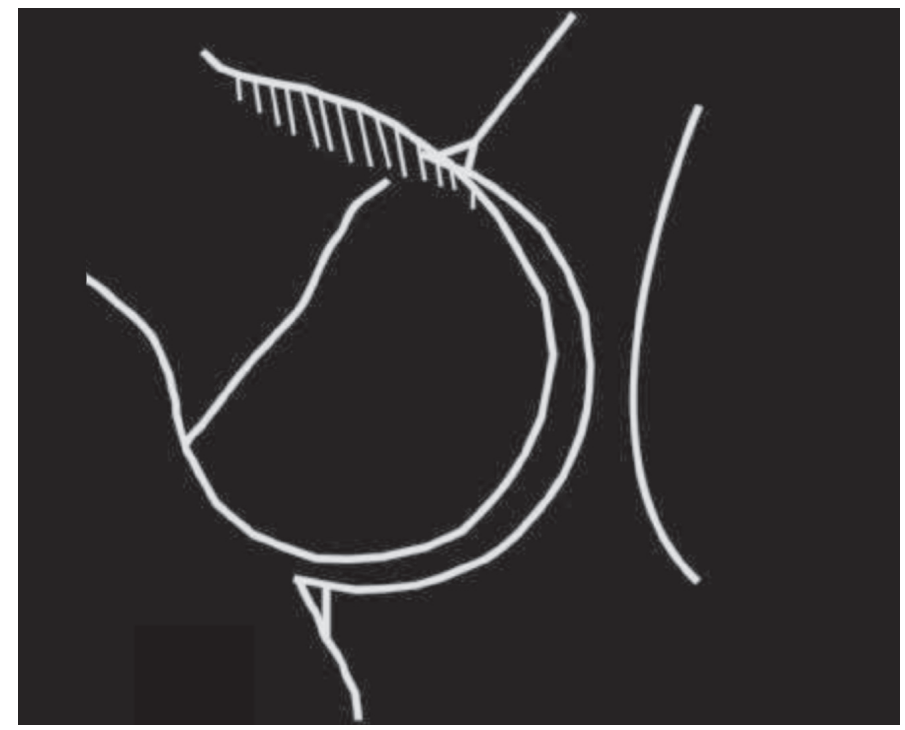

Figura 1 - Representação do impacto tipo came

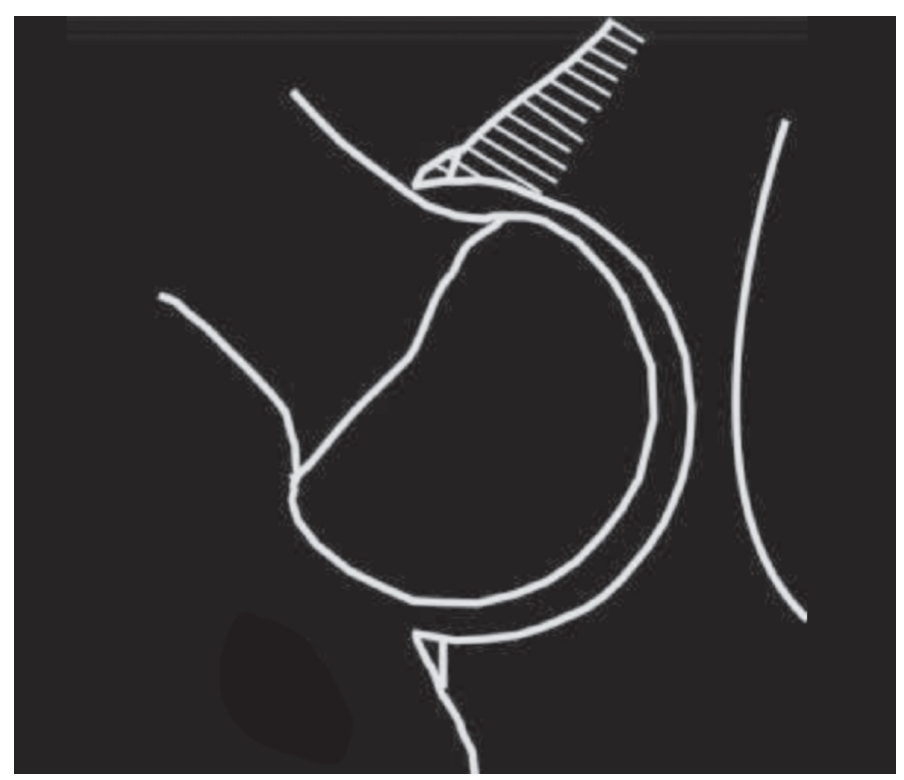

Figura 2 - Representação do impacto tipo pincer

A idade dos pacientes variou de 21 a 55 anos (média de 34 anos). Houve predomínio do sexo masculino em 19 casos (67\%). Quinze pacientes (53\%) praticavam atividade física frequente, sendo 13 de forma amadora (86\%) e dois de forma profissional (14\%). O tempo de queixa pré-operatória variou de seis a 96 meses (média de 26,3 meses). Ao exame físico todos apresentavam dor à flexão de $90^{\circ}$ e rotação interna do quadril.

Quanto à melhora clínica, os pacientes foram avaliados pré e pós-operatoriamente utilizando-se o Harris Hip Score (HHS) modificado por Byrd ${ }^{(14)}$. Foram estratificados em resultados ruins (HHS $<70$ pontos), razoáveis (HHS 70-79), bons (HHS 80-89) e excelentes (HHS 90-100). 
Os pacientes foram avaliados pré e pós-operatoriamente com relação à rotação interna do quadril acometido. Foi realizada mensuração em posição supina com flexão de $90^{\circ}$ do quadril e rotação interna máxima.

Todos os pacientes foram operados e avaliados pelo mesmo cirurgião (GCP).

Os valores obtidos nos índices acima foram analisados estatisticamente através do método de Wilcoxon para a avaliação de variáveis não paramétricas.

Todos os pacientes foram avaliados por radiografias (bacia AP, cross-table, falso perfil de Lequesne) e ressonância nuclear magnética ou artrorressonância magnética quando necessário. Quando realizada artro-RNM, foi injetada marcaína intra-articular como adjuvante na confirmação de etiologia intra-articular da dor. A tomografia computadorizada foi realizada conforme a necessidade, sendo útil para o mapeamento do rebordo acetabular e da transição colo-cabeça femoral. É importante salientar o rigor técnico necessário para a realização das radiografias, pois erros de posicionamento podem afetar a interpretação da versão acetabular e, consequentemente, mascarar ou falsear lesões do tipo pincer.

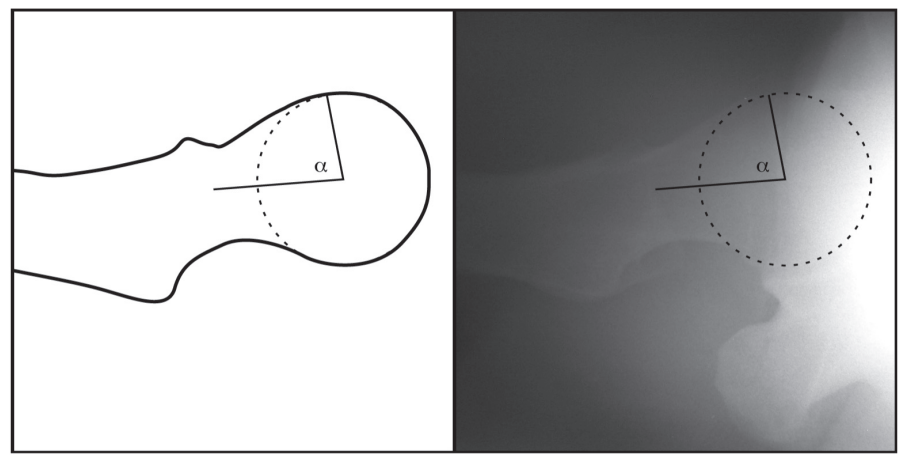

Figura 3 - Radiografia cross-table do quadril direito: diminuição do offset cabeça-colo com ângulo $\alpha$ de $82^{\circ}$

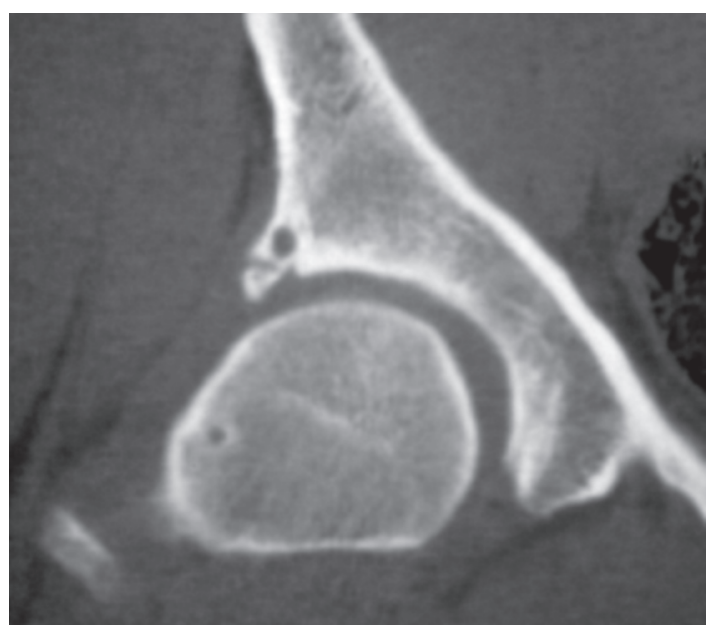

Figura 4 - Corte coronal de tomografia computadorizada do quadril direito, onde se observa cisto perilabial, calcificação da base do lábio acetabular e cisto de impacto no colo femoral

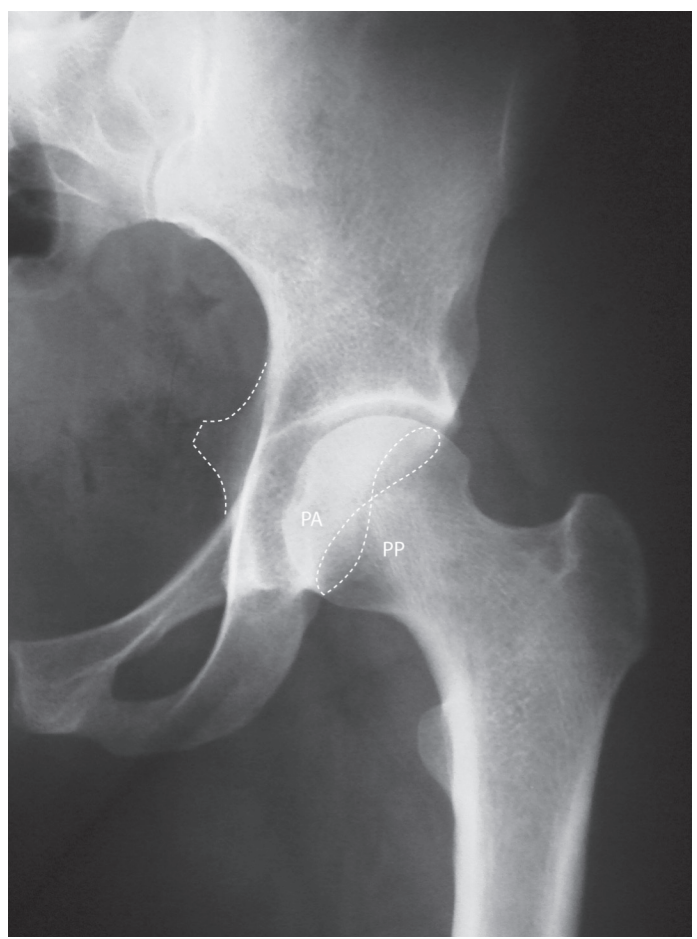

Figura 5 - Radiografia incidência anteroposterior do quadril esquerdo, na qual se visibiliza retroversão acetabular, com sinal do cruzamento e espinha isquiática visível na cavidade pélvica. PA: parede anterior, PP: parede posterior.

Os portais utilizados seguiram as descrições de Philippon e Schenker ${ }^{(15)}$. Geralmente, dois portais foram suficientes: anterior e paratrocantérico anterior. Foram utilizados portais alternativos conforme a necessidade. Ótica de $70^{\circ}$ foi utilizada em todos os casos. Primeiramente, operado o compartimento central, diagnosticando e tratando alterações da cartilagem articular, lesão labial ou lesão do ligamento redondo. O lábio acetabular foi desbridado ou reparado, dependendo do aspecto do tecido labial, localização da lesão ou presença de impacto tipo pincer, sendo preservado sempre que possível. Lesões condrais de espessura total, classificação 4 de Outerbridge, foram tratadas do seguinte modo: se associadas com impacto tipo pincer, o rebordo acetabular foi submetido a osteoplastia, o que geralmente resultou somente em cartilagem de aspecto macroscópico saudável, sem necessidade de microfratura. Caso defeito condral de espessura total permanecesse após a osteocondroplastia ou caso a osteocondroplastia do rebordo não estivesse indicada, foram realizadas microfraturas. Nos casos em que existia lesão total do ligamento redondo, foi realizado desbridamento do mesmo. Posteriormente, foi operado o compartimento periférico, soltando-se a tração, permitindo a passagem da ótica para a região periférica da transição cabeça-colo femoral. Uma vez que a região do impacto tipo came foi definida por alterações morfológicas locais 


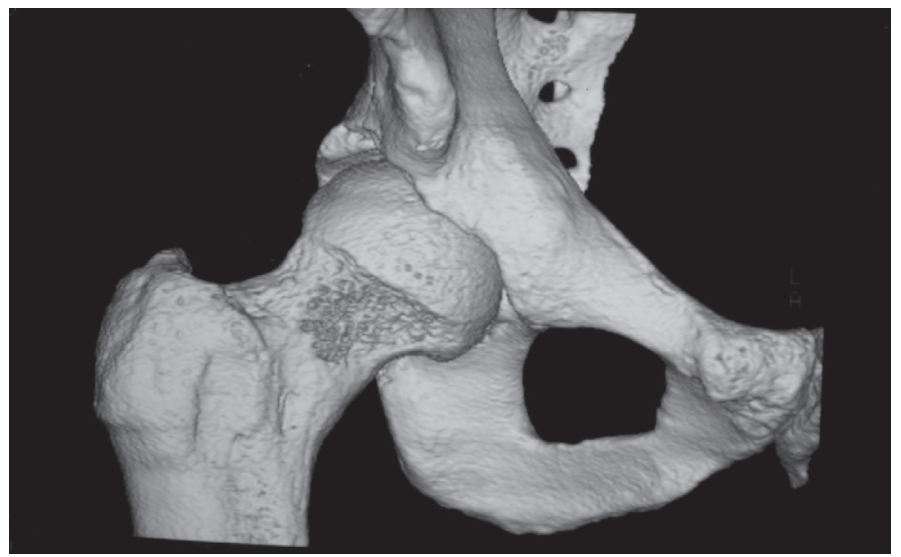

Figura 6

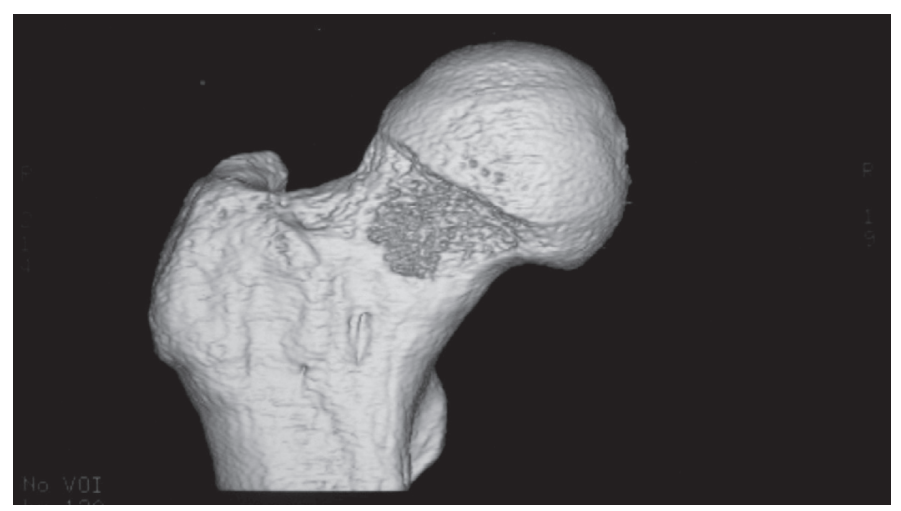

Figura 8

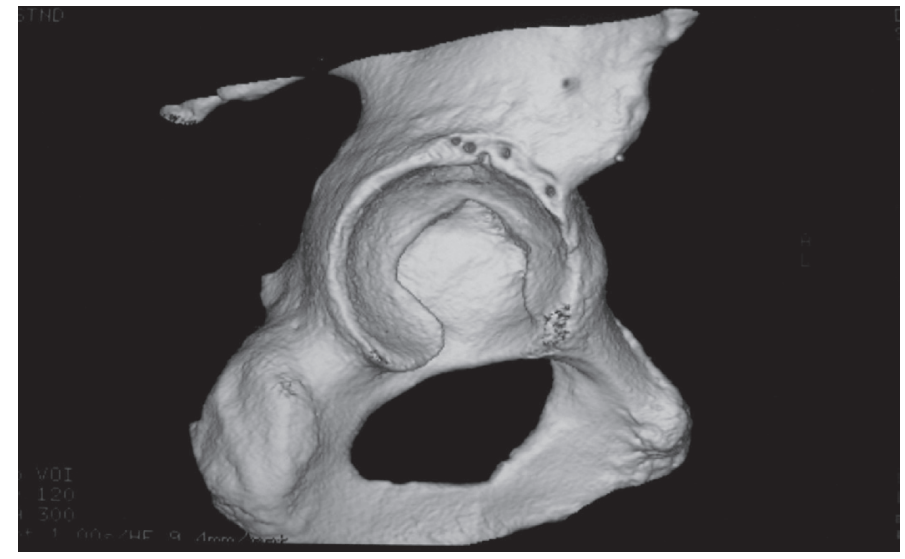

Figura 7

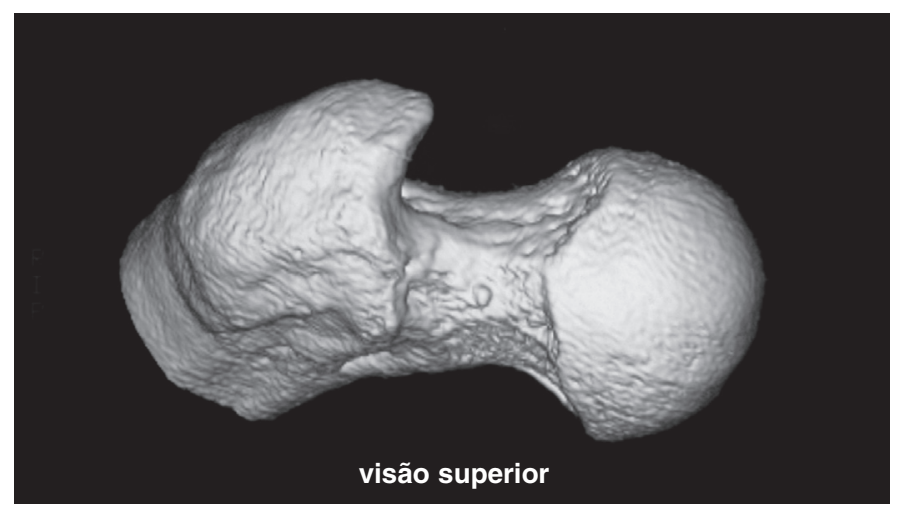

Figura 9

Figuras 6, 7, 8 e 9 - Tomografia computadorizada com reconstrução em três dimensões, onde se observa o resultado da osteocondroplastia femoral e acetabular. Figura 7 - Observam-se orifícios dos locais de colocação das âncoras para refixação labial.

ou exame dinâmico da articulação, foi realizada a osteocondroplastia. Foi considerado o fim do procedimento, quando realizado teste com flexão de $90^{\circ}$, adução e rotação interna de $30^{\circ}$ do quadril e não foi mais visibilizado conflito do colo contra o rebordo acetabular.

\section{RESULTADOS}

O Harris Hip Score médio pré-operatório foi de 58,1 (DP $=13,5$, variando de 22 a 84,7). O HHS médio pósoperatório foi de 96,9 (DP=4,35, variando de 66 a 100). $\mathrm{O}$ aumento médio do HHS pré para o pós-operatório foi de 38,7 pontos.

Os valores do HHS pré e pós-operatórios foram analisados (SPSS versão 10.0, SPSS, Chicago, Ill, EUA). O teste de Wilcoxon foi realizado para análise de variáveis não paramétricas. Encontramos diferença estatisticamente significante entre os valores pré e pós-operatórios do HHS $(\mathrm{P}<0,001)$.

Estratificando os resultados do HHS, tivemos quatro resultados bons $(15 \%)$ e 24 excelentes $(85 \%)$.

Houve um caso de parestesia transitória da região pe- niana, que se resolveu em três semanas. Após a modificação do rolo perineal ${ }^{(16)}$, não houve mais complicações em relação a alterações pós-operatórias do nervo pudendo.

Em relação ao tratamento das lesões labiais, 20 quadris foram submetidos a desbridamento e oito lábios foram reparados com âncoras. Sendo essa uma série consecutiva de pacientes, vale observar que, na grande maioria dos casos recentes, os lábios têm sido reparados/reinseridos.

Todos os pacientes apresentavam pré-operatoriamente limitação da rotação interna do quadril acometido, com rotação interna média de $17^{\circ}(\mathrm{DP}=16,9$, variando de $-15^{\circ}$ a $45^{\circ}$ ). Os pacientes apresentaram aumento da mobilidade em rotação interna do quadril operado, com rotação interna média de $36^{\circ}\left(\mathrm{DP}=11,6\right.$, variando de $0^{\circ}$ a $\left.50^{\circ}\right)$. O aumento médio de rotação interna foi de $19^{\circ}$, variando de 0 a $40^{\circ}$.

O teste de Wilcoxon foi realizado para análise de variáveis não paramétricas. Encontramos diferença estatisticamente significante entre os valores pré e pós operatórios da rotação interna do quadril $(\mathrm{P}<0,001)$.

Não houve nenhum caso de necrose asséptica do quadril, ossificação heterotópica, fratura do colo ou infecção. 


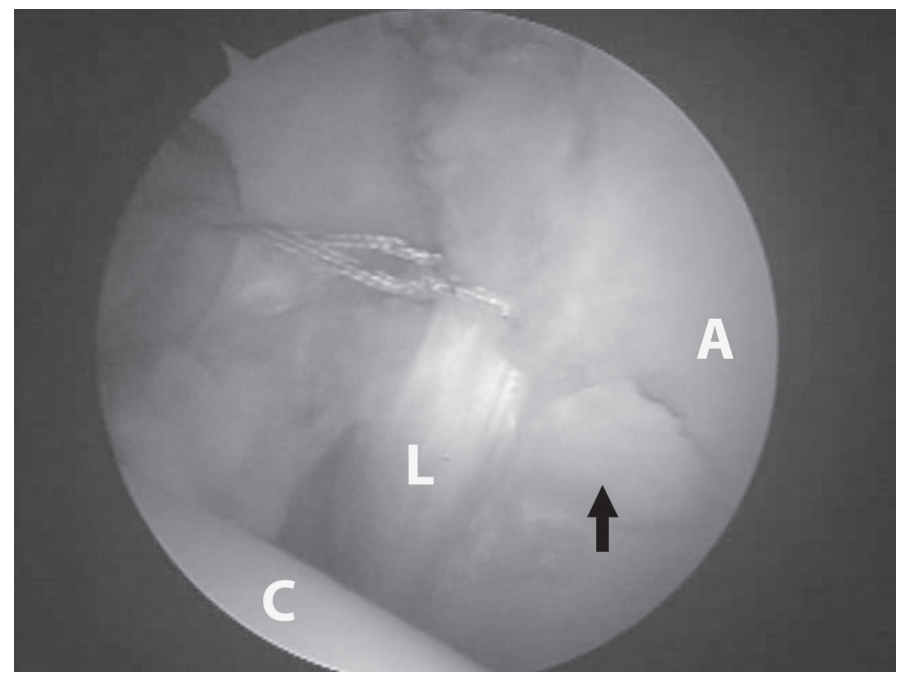

Figura 10 - Refixação labial após osteocondroplastia da sobrecobertura acetabular. Nota-se lesão condral na zona do "divisor de águas" (seta). C: cabeça femoral, L: lábio acetabular, A: cartilagem acetabular. (Imagem gentilmente cedida por Dr. Giancarlo Polesello)

\section{DISCUSSÃO}

Ganz et al, baseados na experiência clinica de mais de 700 luxações cirúrgicas do quadril ${ }^{(4)}$, afirmam que o impacto femoroacetabular gera o desenvolvimento de artrose precoce de quadris não displásicos ${ }^{(5)}$. O conceito é baseado mais no movimento do que na carga axial do quadril. O impacto pode resultar de anormalidades morfológicas que afetam o acetábulo e o fêmur proximal ou pode ocorrer em pacientes que submetem o quadril a amplitudes de movimento extremas e suprafisiológicas. Dependendo da causa subjacente, o impacto femoroacetabular resulta em lesão do lábio e da cartilagem acetabular.

Parâmetros clínicos, radiográficos e intraoperatórios podem ser usados para confirmar o diagnóstico ${ }^{(2,5-8,17)}$. O tratamento cirúrgico do impacto femoroacetabular é baseado na melhora da amplitude de movimento, aliviando o impacto do fêmur contra o rebordo acetabular e suas consequências. Acredita-se que a intervenção cirúrgica precoce, além de aliviar os sintomas, possa desacelerar a progressão do processo degenerativo.

O reconhecimento do impacto femoroacetabular exige e análise cuidadosa de pequenas anormalidades da extremidade proximal do fêmur, assim como anormalidades na orientação ou profundidade do acetábulo, que podem causar dificuldade para o movimento. A rotação interna é o movimento mais critico. Este assunto não é novo. Preiser, em $1911^{(18)}$, já especulava sobre a relação entre a rotação interna reduzida e o desenvolvimento posterior da osteoartrose; entretanto, o conceito moderno de impacto femoroacetabular foi descrito somente há uma década ${ }^{(5)}$.
Embora o impacto femoroacetabular possa localizar-se em qualquer lugar da articulação, o local mais comum é a região anterolateral, sendo produzido pela rotação interna do fêmur em flexão de $90^{\circ}$ do quadril. No teste do impacto anterior, realizado com rotação interna máxima e $90^{\circ}$ de flexão passiva do quadril, observam-se diminuição da rotação interna do quadril e dor associada. Flexão e adução do quadril levam ao conflito do colo femoral com o rebordo acetabular. Rotação interna associada provoca forças cisalhantes no lábio acetabular, similarmente aos meniscos dos joelhos, estimulando as terminações nervosas. Isso provoca dor inguinal aguda em pacientes com o lábio roto ou degenerado ${ }^{(6)}$. Movimentos em alta velocidade são mais destrutivos que o impacto em baixa velocidade, o que torna mais suscetíveis os atletas ou pacientes que praticam atividade física extenuante ${ }^{(15)}$.

Dois tipos distintos de impacto femoroacetabular foram identificados ${ }^{(5)}$, sendo frequentemente combina$\operatorname{dos}^{(7)}$. O primeiro é caracterizado por impacto linear do rebordo acetabular contra a junção cabeça-colo femoral devido a sobrecobertura acetabular, chamado tipo pincer. O segundo tipo ocorre com a compressão da extensão não esférica da cabeça femoral na cavidade acetabular, chamado tipo came.

O impacto de causas femorais (idiopática, epifisiólise, retroversão pós- traumática da cabeça femoral, deformidade secundária a doença de Perthes ou necrose avascular da cabeça femoral) é chamado impacto tipo came, tendo sido descrito em 1968 por Carlioz et $\mathrm{al}^{(3)}$. O setor não esférico da cabeça ou da junção cabeça-colo é comprimido dentro do acetábulo provocando abrasão de "fora para dentro" ou avulsão da cartilagem articular, enquanto o lábio num primeiro momento permanece intacto $^{(2)}$. Com o tempo, a substância do lábio desinserido degenera, enquanto a região da borda lateral permanece com sua textura normal por longo período ${ }^{(6)}$, permitindo sua refixação ${ }^{(19)}$. A preservação de qualquer porção sadia do lábio é importante devido ao fato de o lábio no quadril normal funcionar como lubrificador da articulação, distribuidor de pressão e absorvedor de choques. $\mathrm{O}$ sucesso da refixação já foi comprovado por artro-RNM e artroscopias. A cartilagem que recobre a parte esférica da cabeça permanece intacta por um longo período, ao contrário da cartilagem da região não esférica, que mostra sinais degenerativos progressivos ${ }^{(2)}$.

O impacto de causas acetabulares (retroversão idiopática e de outros tipos, coxa profunda e protrusão acetabular) é chamado impacto tipo pincer. Existem evidências de que a retroversão idiopática é mais uma alteração patomorfológica pélvica do que acetabular ${ }^{(17)}$. Com a retroversão do acetábulo, a espinha isquiática torna-se visível 
na cavidade pélvica na maioria dos casos. $\mathrm{O}$ impacto do tipo pincer é produzido por colisão do tipo linear entre a junção cabeça-colo femoral e a área de sobre cobertura acetabular. $\mathrm{O}$ fato de o lábio ser espremido e conter terminações nervosas pode explicar por que esse tipo de impacto, frequentemente encontrado em mulheres, é mais doloroso que o tipo came. Com o impacto crônico do lábio acetabular ocorre degeneração e formação de cistos intrassubstanciais. O mecanismo de irritação crônica pode provocar aposição óssea no rebordo acetabular que, por crescimento progressivo, provoca afilamento do lábio e aumenta a sobrecobertura, piorando o impacto ${ }^{(2)}$.

A cabeça femoral permanece intacta por longo período e tardiamente ocorrerá abrasão da cartilagem na região articular póstero-inferior na cabeça e/ou no acetábulo, ocorrendo alterações chamadas "lesões em contragolpe", resultando posteriormente em artrose pósteromedial do quadril ${ }^{(7)}$. Embora mais doloroso, o impacto tipo pincer provoca destruição menor e mais lenta da cartilagem articular.

O impacto do tipo pincer é encontrado mais comumente em mulheres, entre 30 e 40 anos, que realizam atividades com grande amplitude de movimento. $\mathrm{O}$ impacto do tipo came é tipicamente encontrado em homens, entre 20 e $30 \operatorname{anos}^{(2)}$. Beck et $a l^{(7)}$ relatam que a maioria dos casos é uma mistura desses dois tipos.

O tratamento conservador pode até ser tentado inicialmente, com restrição de atividades atléticas e uso de antiinflamatórios não hormonais. Fisioterapia com objetivo de ganho de amplitude de movimento ou alongamento podem resultar em piora dos sintomas, principalmente se orientado a flexão e adução do quadril. O tratamento pode ter sucesso temporário devido à idade jovem desses pacientes, porém, dado o alto nível de atividade física e esportiva, geralmente falha. Por tratar-se de um problema anatômico, que comprovadamente leva à destruição progressiva do lábio e da cartilagem articular e consequente artrose, o tratamento cirúrgico deve ser realizado precocemente na falha do tratamento conservador.

O tratamento do impacto não é assunto novo. SmithPetersen, em 1936, já descreveu o impacto femoroacetabular, inclusive seu tratamento com osteoplastia acetabular e femoral ${ }^{(20)}$. Seus maus resultados talvez ocorressem por ter realizado tais intervenções em quadris com artrose avançada. Sabe-se hoje que isso não tende a propiciar bons resultados.

$\mathrm{O}$ tratamento cirúrgico do impacto femoroacetabular consiste no remodelamento do fêmur proximal, osteoplastia ou reorientação da sobrecobertura acetabular e reparo/refixação labral ou desbridamento. Várias técnicas cirúrgicas foram descritas para o tratamento do impacto femoroacetabular, sendo elas: osteotomia periacetabu$\operatorname{lar}^{(8)}$, técnica de luxação cirúrgica do quadril ${ }^{(4)}$, artroscopia com osteocondroplastia via pequena incisão ${ }^{(21)}$, técnica via anterior modificada ${ }^{(22)}$ e $\operatorname{artroscopia}^{(15,23)}$.

O tipo de abordagem deve ser escolhido de acordo com a complexidade da morfologia do impacto femoroacetabular e o treinamento do cirurgião.

A artroscopia de quadril já está presente em nosso meio há mais de 15 anos $^{(24)}$, porém, seu uso para o tratamento do IFA é recente. Avanços nas técnicas artroscópicas, particularmente na incorporação da avaliação dinâmica intraoperatória do impacto, permitiu o IFA ser abordado de forma menos invasiva. A anatomia artroscópica já foi extremamente estudada, estabelecendo-se portais artroscópicos bem definidos e seguros ${ }^{(25)}$ e técnica anatômica em relação à preservação da vascularização do colo femo$\mathrm{ral}^{(26)}$. O reparo labial é possível por via $\operatorname{artroscópica~}^{(27)}$. O cirurgião deve estar familiarizado com a anatomia artroscópica, pois pode haver dificuldade de orientação quanto à localização e quantidade necessária de ressecção óssea, podendo levar a correção insuficiente e consequente impacto residual ou até ressecção excessiva, que é associada com risco de fratura colofemoral ou instabilidade ${ }^{(28)}$. Estudos anatômicos comprovaram a mesma precisão da osteoplastia do colo por via aberta e artroscópica por cirurgiões treinados ${ }^{(29)}$, assim como estudos clínicos demonstraram a eficácia da restauração do offset femoral por via artroscópica $^{(30)}$. Desse modo, acreditamos que existem subsídios para afirmar que a técnica de tratamento artroscópico do IFA é factível e reprodutível.

Siebenrock et al $^{(8)}$ avaliaram 29 quadris submetidos à osteotomia periacetabular devido à retroversão acetabular, com seguimento médio de 30 meses, com resultados bons e excelentes em 26 quadris (89\%).

Em relação ao tratamento por luxação cirúrgica, Beck et $a l^{(10)}$ obtiveram resultados excelentes em $68 \%$ dos casos. Murphy et al ${ }^{(11)}$ observaram que $65 \%$ dos pacientes operados, com seguimento de dois a 12 anos, não necessitaram de novas intervenções. Peters e Erickson $^{(12)}$ avaliaram 30 pacientes, o HHS melhorou de uma média de 70 no pré-operatório para 87 no pós-operatório mais recente. Parvizi et al ${ }^{(31)}$ relatam $76 \%$ dos pacientes com resultados excelentes ou bons, com média de seguimento de 4,7 anos.

Zebala et $a l^{(32)}$, utilizando a técnica artroscópica com osteocondroplastia via pequena incisão, com seguimento médio de 1,5 anos, relataram HHS pré-operatório médio de 63,8 e pós-operatório de 92,3 , totalizando $95,8 \%$ de resultados bons e excelentes.

Ribas et $a l^{(22)}$ relatam melhora da dor em todos os casos utilizando a via de Smith-Petersen modificada. 
Quanto ao tratamento artroscópico, Larson e Giveans $^{(33)}$ avaliaram o tratamento de 47 pacientes com seguimento mínimo de três meses (média de 9,9 meses): 26 quadris $(55,3 \%)$ foram avaliados como excelentes, nove $(19.1 \%)$ como bons, cinco $(10,6 \%)$ como razoáveis e sete $(14.9 \%)$ como ruins. O HHS modificado pré-operatório médio foi de 60,4, 72,8 em seis semanas, 81,6 em três meses, 83,1 em seis meses e 85,4 em um ano. Philippon et $a l^{(34)}$ avaliaram 45 atletas profissionais, todos com alivio dos sintomas e retorno aos esportes. Sampson $^{(23)}$ avaliou 320 pacientes, $90 \%$ com eliminação do sinal do impacto e satisfeitos com o resultado. Ilizaturri et $a l^{(35)}$ avaliaram 19 pacientes com impacto tipo came, com mínimo de dois anos de seguimento; $84 \%$ deles tiveram melhora dos sintomas.

Nossos resultados estão de acordo com as séries da literatura que apresentam média de $75 \%$ a $95 \%$ de bons resultados. Em nossa série, o Harris Hip Score médio pré-operatório foi de 58,1 e o pós-operatório, 96,9, com aumento médio do HHS de 38,7 pontos, resultados também de acordo com a literatura. O que aprendemos é que a indicação minuciosa dos casos é o fator fundamental para um bom resultado, já que casos mal-indicados levam a resultados ruins. A partir dos resultados de varias séries ${ }^{(8,10,23,36)}$, observou-se que pacientes com sinais radiográficos de artrose não são bons candidatos a cirurgias preservadoras.

Em uma comparação de estudos clínicos quanto à amplitude da rotação interna no impacto femoroacetabular, Eijer et $a l^{(37)}$ avaliaram nove pacientes com impacto tipo came pós-traumático; apresentavam pré-operatoriamente rotação interna média de $7^{\circ}$, com aumento médio de $9^{\circ}$ apos ressecção da excrescência óssea por via aberta. Siebenrock et al ${ }^{(8)}$ avaliaram 29 pacientes com impacto tipo pincer, com rotação interna média pré-operatória de $11^{\circ}$, submetidos à osteotomia periacetabular com média pós-operatória de $21^{\circ}$. Leunig et al ${ }^{(38)}$ avaliaram 14 pacientes com impacto femoroacetabular não especificado que apresentavam rotação interna pré-operatória média de $15^{\circ}\left(\mathrm{DP}=12^{\circ}\right)$. Os autores não informaram nessa série a mobilidade pós-operatória. Jager et al ${ }^{(8)}$ avaliaram 17 pacientes com impacto do tipo came, com rotação interna média pré-operatória de $8^{\circ}$, submetidos à ressecção da excrescência óssea por via aberta com média pós-operatória de $21,7^{\circ}$. Strehl e Ganz ${ }^{(39)}$ avaliaram 11 pacientes com impacto tipo came pós-traumático, que apresentavam pré-operatoriamente rotação interna média de $15^{\circ}$, submetidos à ressecção da excrescência óssea via aberta com média pós-operatória de $20^{\circ}$. Wettstein e Dienst ${ }^{(40)}$ avaliaram 15 pacientes com impacto tipo misto que apresentavam rotação interna pré-operatória de $7^{\circ}\left(\mathrm{DP}=12^{\circ}\right)$; foram submetidos a artroscopia e não foi informada mobilidade pós-operatória. Em uma simulação computadorizada da mobilidade articular adquirida através de tomografia computadorizada, o ganho médio de rotação interna na correção de impacto tipo pincer foi de $5,4^{\circ}$, no impacto came de $8,5^{\circ}$, e no impacto do tipo misto de $15,7^{\circ(41)}$. Ribas et al ${ }^{(22)}$ avaliaram 14 pacientes, com rotação interna média pré-operatória de $-17^{\circ}\left(-14^{\circ}\right.$ a $\left.-28^{\circ}\right)$ submetidos à osteocondroplastia por via anterior, com média pós-operatória de $23^{\circ}\left(14^{\circ} \mathrm{a}\right.$ $\left.32^{\circ}\right)$. Stähelin et al(30) avaliaram 23 pacientes com impacto tipo came com rotação interna média pré-operatória de $5^{\circ}$, submetidos a correção artroscópica com média pós-operatória de $22^{\circ}$. O ganho médio de rotação interna pós-operatório foi de $19^{\circ} \mathrm{em}$ nossa série, o que está de acordo com a literatura. A maioria dos casos de nossa série foi de impacto do tipo misto.

Existem algumas limitações neste estudo. Uma é a falta de grupo controle para a avaliação dos resultados do tratamento. Outra é literatura suficiente para permitir comparação dos resultados, inclusive com a técnica aberta. Existe um único estudo (preliminar) prospectivo comparando o tratamento aberto e artroscópico, realizado por Sadri e Hoffmeyer ${ }^{(42)}$, onde foram demonstrados bons resultados em ambas as técnicas, sugerindo que o tratamento artroscópico possa ser vantajoso por evitar as complicações da cirurgia aberta. Muitas das séries publicadas não utilizam os mesmos critérios de avaliação funcional pós-operatória. O HHS é uma ótima ferramenta para avaliação funcional, porém, outros autores estão desenvolvendo novos escores clínicos.

A magnitude da melhora de nossos pacientes é consistente com a de outras séries de tratamento aberto e artroscópico.

Outro ponto que vale a pena ser discutido é a reparação labial. Tannast et $a l^{(43)}$ realizaram um estudo correlacionando achados intra-operatórios com simulações computadorizadas da região de maior impacto femoroacetabular. Foi observada associação entre a presença de lesões labiais e a degeneração da superfície articular adjacente, o que é principalmente verificado nos procedimentos artroscópicos ${ }^{(44)}$. Entretanto, o que todos esses estudos têm em comum é que eles falham em uma explicação satisfatória para a causa da lesão articular. A maioria dos autores descreve um trauma direto durante atividade esportiva como a etiologia de lesões labiais. De fato, elas raramente acontecem na ausência de alterações morfológicas ósseas ${ }^{(45)}$. Portanto, os resultados artroscópicos somente do desbridamento 
labial sem tratamento da alteração anatômica subjacente (na maioria dos casos de IFA) são insatisfatórios ${ }^{(46)}$. Em nossa opinião, a grande maioria dos casos de lesões labiais está associada ao impacto femoroacetabular, sendo o lábio acetabular a primeira estrutura a falhar ${ }^{(6)}$.

A degeneração labial tem sido observada em casos de $\mathrm{OA}^{(47)}$. Ikeda et al ${ }^{(48)}$ postularam que lesões do lábio acetabular podem comprometer suas funções de absorção de carga e estabilização, levando a artrose, similarmente ao encontrado em lesões meniscais. Estudos de elementos finitos demonstraram que, ao comprometer as funções seladoras do lábio, aumentam-se o estresse cartilaginoso e forças cisalhantes, que podem contribuir para a lesão por fadiga da cartilagem adjacente. McCarthy et al ${ }^{(44)}$ também observaram associação entre lesões labiais e da cartilagem adjacente, sugerindo que as lesões labiais e a osteoartrose são um contínuo da doença.

Devido às maiores forças na articulação do quadril, a refixação labial pode ser desafiadora. Entretanto, mesmo com o desenvolvimento de um mecanismo de ancoragem suficientemente forte, a capacidade de regeneração biológica da matriz labral é necessária. O que nos leva a ter boas expectativas é que Ito et $a l^{(6)}$ observaram que, mesmo em casos avançados de artrose, a ponta do lábio, local de sua irrigação, permanece preservada, o que permite seu reparo.

Byrd e Jones ${ }^{(36)}$ avaliaram 30 quadris submetidos a tratamento de lesão labial com desbridamento. As lesões labiais foram desbridadas até ficar estáveis. Após 10 anos de seguimento, os pacientes sem sinal de artrose tiveram $82 \%$ de bons resultados,com o HHS pré-operatório médio de 54 e pós-operatório de 90 . Referem que $88 \%$ dos pacientes com artrose tiveram que ser convertidos para PTQ.

Espinosa et $a l^{(19)}$ avaliaram o efeito do desbridamento do lábio comparado com o reparo/refixação e encontraram melhores resultados no seguimento de dois anos no grupo submetido a reparo labial em relação a dor e progressão da artrose. Não está claro se os melhores resultados vieram de uma técnica mais apurada ou da preservação labial, pois essa é uma série consecutiva de pacientes.

Baseados em todas essas afirmações, embora ainda não existam observações clínicas suficientes para afirmar que a reparação labial leva a melhores resultados, acreditamos que esta é justificada. Existe a necessidade de estabelecerem-se critérios para a possível preservação/reparo labial. Nossos resultados necessitam de maior tempo de seguimento para comparar-se a significância dos resultados nos diferentes tratamentos.

O seguimento mínimo utilizado para a avaliação de nossa série foi de um ano. Na série de Larson e Give- ans ${ }^{(33)}$, a análise do HHS revelou melhora nos resultados, porém, sem melhora significativa após três meses de seguimento; pode-se interpretar que talvez três meses de seguimento sejam tempo mínimo suficiente para avaliar a melhora dos pacientes. Por outro lado, Sampson ${ }^{(22)}$ avaliou 158 pacientes e encontrou melhora de $50 \%$ da dor em três meses, $75 \%$ em cinco meses e $95 \%$ em um ano. Não havia dados suficientes em nossa série para avaliar essa progressão. Por isso optamos pelo seguimento mínimo de um ano. Novos dados permitirão avaliar se realmente existe um período de melhora até dois anos, quando haveria nova queda funcional nos pacientes com mau prognóstico (Byrd, comunicação pessoal).

Segundo a literatura ${ }^{(49-50)}$, a incidência de complicações varia de 0,5 a $5 \%$ dos casos, sendo mais comum a neuropraxia devido à tração. Em uma análise de nossos dados $^{(16,50)}$, a maior parte das complicações ocorreu pela tração (3,6\%). Após a modificação do rolo perineal, não houve mais complicações em relação a alterações pósoperatórias do nervo pudendo. Nossos índices estão de acordo com o relatado na literatura.

O tratamento por via aberta do IFA é considerado o padrão, porém, como aconteceu primeiramente com a artroscopia do joelho e posteriormente com a do ombro, uma série de lesões passou a ser tratada por via artroscópica. Cuidado é necessário para impedir um crescimento descontrolado dos procedimentos artroscópicos do quadril, evitando o fenômeno que ocorreu na cirurgia abdominal, onde houve aumento do número de colecistectomias com a introdução da laparoscopia. Do mesmo modo, é necessário estabelecer-se que padrões de lesão têm seu tratamento melhor alcançado por via aberta ou artroscópica.

Concluindo, os resultados do tratamento do impacto femoroacetabular têm sido promissores na ausência de lesão condral significativa no momento da cirurgia. $\mathrm{O}$ prognóstico do quadril é significativamente melhor se o impacto é eliminado precocemente ${ }^{(9)}$; portanto, a cirurgia deve ser recomendada assim que os primeiros sintomas surgirem $^{(10,11)}$. Persiste a dúvida do que fazer nos casos que têm impacto definido, porém assintomáticos ou que melhoram completamente com o tratamento conservador. Nossa conduta é aguardar e operar somente pacientes sintomáticos até que evidências definitivas surjam. Resta a dúvida quanto à perenidade da melhora da dor e o possível retardo no desenvolvimento de artrose.

\section{CONCLUSÃO}

O tratamento artroscópico do impacto femoroacetabular tem resultados satisfatórios. 


\section{REFERÊNCIAS}

1. Murray RO. The aetiology of primary osteoarthritis of the hip. $\mathrm{Br} \mathrm{J}$ Radiol. 1965;38(455):810-24.

2. Ganz R, Leunig M, Leunig-Ganz K, Harris WH. The etiology of osteoarthritis of the hip: an integrated mechanical concept. Clin Orthop Relat Res. 2008;466(2): 264-72.

3. Carlioz H, Pous JG, Rey JC. Les epiphysiolyses femorales superieures. Rev Chir Orthop Reparatrice Appar Mot. 1968,54(5):387-491.

4. Ganz R, Gill TJ, Gautier E, Ganz K, Krügel N, Berlemann U. Surgical dislocation of the adult hip a technique with full access to the femoral head and acetabulum without the risk of avascular necrosis. J Bone Joint Surg Br. 2001;83(8):1119-24.

5. Ganz R, Parvizi J, Beck M, Leunig M, Nötzli H, Siebenrock KA. Femoroacetabular impingement: a cause for osteoarthritis of the hip. Clin Orthop Relat Res. 2003;(417):112-20.

6. Ito K, Leunig M, Ganz R. Histopathologic features of the acetabular labrum in femoroacetabular impingement. Clin Orthop Relat Res. 2004;(429):262-71

7. Beck M, Kalhor M, Leunig M, Ganz R. Hip morphology influences the pattern of damage to the acetabular cartilage: femoroacetabular impingement as a cause of early osteoarthritis of the hip. J Bone Joint Surg Br. 2005;87(7):1012-8.

8. Siebenrock KA, Schoeniger R, Ganz R. Anterior femoro-acetabular impingement due to acetabular retroversion. Treatment with periacetabular osteotomy. J Bone Joint Surg Am. 2003;85-A(2):278-86.

9. Crestani MV, Teloken MA, Gusmão PDF. Impacto femoroacetabular: uma das condições precursoras da osteoartrose do quadril. Rev Bras Ortop. 2006;41(8):285-93

10.Beck M, Leunig M, Parvizi J, Boutier V, Wyss D, Ganz R. Anterior femoroacetabular impingement: part II. Midterm results of surgical treatment. Clin Orthop Relat Res. 2004;(418):67-73.

11. Murphy S, Tannast M, Kim YJ, Buly R, Millis MB. Debridement of the adult hip for femoroacetabular impingement: indications and preliminary clinical results. Clin Orthop Relat Res.2004;(429):178-81.

12.Peters CL, Erickson JA. Treatment of femoro-acetabular impingement with surgical dislocation and debridement in young adults. J Bone Joint Surg Am. 2006;88(8):1735-41.

13.Busse J, Gasteiger W, Tönnis D. Eine neue methode zur röntgenologischen beurteilung eines hüftgelenkes - der hüftwert. Arch Orthop Unfallchir. 1972;72(1):1-9.

14. Byrd JW. Avoiding the labrum in hip arthroscopy. Arthroscopy. 2000; 16(7):770-3.

15.Philippon MJ, Schenker ML. Arthroscopy for the treatment of femoroacetabular impingement in the athlete. Clin Sports Med. 2006; 25(2):299-308.

16.Polesello GC, Honda EK, Souza BGC, Guimarães RP, Junior WR, Ono NK. Poste Perineal modificado para artroscopia de quadril: um equipamento mais seguro para tração articular. In: $12^{\circ}$ Congresso de Ortopedia e Traumatologia do Estado de São Paulo (COTESP). Campinas, SP, 2008. São Paulo: Sociedade Brasileira de Ortopedia e Traumatologia; 2008.

17.Jamali AA, Mladenov K, Meyer DC, Martinez A, Beck M, Ganz R, et al. Anteroposterior pelvic radiographs to assess acetabular retroversion: high validity of the "cross-over-sign". J Orthop Res. 2007;25(6):758-65

18.Preiser G. Statische Gelenkerkrankungen. Enke, Stuttgart; 1911. p.78

19. Espinosa N, Rothenfluh DA, Beck M, Ganz R, Leunig M. Treatment of femoroacetabular impingement: preliminary results of labral refixation. $\mathrm{J}$ Bone Joint Surg Am. 2006;88(5):925-35.

20.Smith-Petersen MN. Treatment of malum coxae sinilis, old slipped upper femoral epiphysis, intrapelvic protusion of the acetabulum, and coxa plana by means of acetabuloplasty. J Bone Joint Surg Am. 1936; 18:869-80.

21. Clohisy JC, McClure JT. Treatment of anterior femoroacetabular impingement with combined hip arthroscopy and limited anterior decompression. lowa Orthop J. 2005;25:164-71.

22. Ribas M, Ginebreda I, Candioti L, Vilarrubias JM. Surgical treatment of the anterior femoroacetabular impingement syndrome of the hip. J Bone Joint Surg Br. 2005; 87(Suppl 1):84.

23. Sampson TG. Arthroscopic treatment of femoroacetabular impingement: a proposed technique with clinical experience. Instr Course Lect. 2006;55:337-46.

24.Lage LA, Costa RC. Artroscopia do quadril: indicações e técnica. Rev Bras Ortop. 1995;30(8):555-8.

25. Robertson WJ, Kelly BT. The safe zone for hip arthroscopy: a cadaveric assessment of central, peripheral, and lateral compartment portal placement. Arthroscopy. 2008;24(9):1019-26

26. Sussmann PS, Ranawat AS, Shehaan M, Lorich D, Padgett DE, Kelly BT. Vascular preservation during arthroscopic osteoplasty of the femoral head-neck junction: a cadaveric investigation. Arthroscopy. 2007;23(7):738-43.
27. Kelly BT, Weiland DE, Schenker ML, Philippon MJ. Arthroscopic labral repair in the hip: surgical technique and review of the literature. Arthroscopy. 2005;21(12):1496-504.

28. Mardones RM, Gonzalez C, Chen Q, Zobitz M, Kaufman KR, Trousdale RT. Surgical treatment of femoroacetabular impingement: evaluation of the effect of the size of the resection. J Bone Joint Surg Am. 2005;87(2):273-9.

29.Sussmann PS, Ranawat AS, Lipman J, Lorich DG, Padgett DE, Kelly BT. Arthroscopic versus open osteoplasty of the head-neck junction: a cadaveric investigation. Arthroscopy. 2007;23(12):1257-64.

30.Stähelin L, Stähelin T, Jolles BM, Herzog RF. Arthroscopic offset restoration in femoroacetabular cam impingement: accuracy and early clinical outcome. Arthroscopy, 2008;24(1):51-7.

31.Parvizi J, Ganz R, Beck M, Leunig M, Sibenrock K. Femoroacetabular impingement: mid-term clinical results. Abstracts from the AAHKS Thirteenth Annual Meeting. J Arthroplasty. 2004;19:261-2.

32.Zebala L, Clohisy JC, Hinkle S, McClure JT, Robison JF. Adult reconstruction hip 5: nonarthroplasty operative treatments, economic issues and miscellaneous. Combined hip arthroscopy and limited open osteochondroplasty for treating impingement disease. [Podium 468]. In: 2007 Annual Meeting Podium Presentations. San Diego: American Academy of Orthopaedic Surgeons, American Association of Orthopaedic Surgeon; 2007.

33.Larson CM, Giveans MR. Arthroscopic management of femoroacetabular impingement: early outcomes measures. Arthroscopy. 2008;24(5):540-6.

34.Philippon M, Schenker M, Briggs K, Kuppersmith D. Femoroacetabular impingement in 45 professional athletes: associated pathologies and return to sport following arthroscopic decompression. Knee Surg Sports Traumatol Arthrosc. 2007;15(7):908-14.

35. Ilizaliturri VM Jr, Orozco-Rodriguez L, Acosta-Rodríguez E, Camacho-Galindo J. Arthroscopic treatment of cam-type femoroacetabular impingement. Arthroplasty. 2008;23(2):226-34.

36.Byrd JWT, Jones KS. Hip arthroscopy for labral pathology: prospective analysis with 10-year follow-up (SS-57). Arthroscopy.2008;24(6):e32-e33.

37. Eijer H, Myers SR, Ganz R. Anterior femoroacetabular impingement after femoral neck fractures. J Orthop Trauma. 2001;15(7):475-81.

38. Leunig M, Podeszwa D, Beck M, Werlen S, Ganz R. Magnetic resonance arthrography of labral disorders in hips with dysplasia and impingement. Clin Orthop Relat Res. 2004;(418):74-80.

39. Strehl A, Ganz R. Anterior femoroacetabular impingement after healed femoral neck fractures. Unfallchirurg. 2005;108(4):263-73.

40.Wettstein M, Dienst M. Hip arthroscopy for femoro-acetabular impingement. Orthopade. 2006;35(1):85-93.

41. Kubiak-Langer M, Tannast M, Murphy SB, Siebenrock KA, Langlotz, F. Range of motion in anterior femoroacetabular impingement. Clin Orthop Relat Res. 2007; 458:117-24.

42. Sadri $\mathrm{H}$, Hoffmeyer P. Treatment of femoroacetabular impingement by hip arthroscopy compared to open surgery with a minimum 2 year follow-up. In: Advances in Hip Arthroscopy Meeting. Paris; 2006.

43. Tannast M, Goricki D, Beck M, Murphy SB, Siebenrock KA. Hip damage occurs at the zone of femoroacetabular impingement. Clin Orthop Relat Res. 2008;(466):273-80.

44. McCarthy JC, Noble PC, Schuck MR, Wright J, Lee J. The Otto E. Aufranc Award: The role of labral lesions to development of early degenerative hip disease. Clin Orthop Relat Res. 2001;(393):25-37.

45.Wenger DE, Kendell KR, Miner MR, Trousdale RT. Acetabular labral tears rarely occur in the absence of bony abnormalities. Clin Orthop Relat Res. 2004;(426):145-50.

46. Kim KC, Hwang DS, Lee CH, Kwon ST. Influence of femoro-acetabular impingement on results of hip arthroscopy in patients with early osteoarthritis. Clin Orthop Relat Res. 2007;(456):128-32.

47.Lage LA, Costa RC. Villar RN. A importância do "labrum" acetabular: revisão da literatura. Rev Bras Ortop. 1996;31(10):792-6.

48. Ikeda T, Awaya G, Suzuki S, Okada Y, Tada H. Torn acetabular labrum in young patients: arthroscopic diagnosis and management. J Bone Joint Surg Br. 1988;70(1):13-6.

49. Clarke MT, Arora A, Villar RN. Hip arthroscopy: complications in 1054 cases. Clin Orthop Relat Res. 2003;(406):84-8.

50.Polesello GC, Honda EK, Souza BGC, Dani WS, Guimarães RP, Junior WR, et al. Complicações em artroscopia do quadril. In: $12^{\circ}$ Congresso de Ortopedia e Traumatologia do Estado de São Paulo (COTESP). Campinas, SP, 2008. São Paulo: Sociedade Brasileira de Ortopedia e Traumatologia; 2008. 